\title{
Impact of Lin28 on lymph node metastasis in papillary thyroid carcinoma
}

\author{
SHUXUN JIN $^{1}$, CHAOYANG XU ${ }^{1}$, LINBO WANG ${ }^{2}$, JIANGGUO WEI $^{3}$ and SONGXIANG WANG ${ }^{2}$ \\ ${ }^{1}$ Department of Breast and Thyroid Surgery, Affiliated Jiunhua Hospital, Zhejiang University School of Medicine, Jiunhua, \\ Zhejiang 321000; Departments of ${ }^{2}$ Breast and Thyroid Surgery and ${ }^{3}$ Pathology, Shaoxing People's Hospital, \\ Shaoxing Hospital, Zhejiang University School of Medicine, Shaoxing, Zhejiang 312000, P.R. China
}

Received February 6, 2020; Accepted September 23, 2020

DOI: $10.3892 / 01.2020 .12358$

\begin{abstract}
Lin28 is involved in the progression of several types of tumors. Data collected from clinical trials have suggested that Lin 28 expression is correlated with poor prognosis in thyroid carcinoma. The present study was conducted to investigate the association between Lin28 expression and the clinicopathological parameters of papillary thyroid carcinoma (PTC). Accordingly, the clinical data and diagnostic results from 237 patients with PTC were collected. Immunohistochemical staining was performed to evaluate the Lin 28 expression levels in thyroid tissue samples. Associations between the expression levels and clinicopathological parameters were evaluated. Lin 28 was expressed in 96/237 (40.5\%) of PTC specimens. Compared with patients with no Lin 28 expression, patients with expression had higher rates of lymph node metastasis $(\mathrm{P}<0.001)$ and larger tumors $(\mathrm{P}=0.011)$. Multivariate analysis revealed that $\operatorname{Lin} 28$ was associated with lymph node metastasis. Next, bioinformatics analysis was performed based using the Gene Expression Omnibus database and The Cancer Genome Atlas database. Lin28 expression was associated with aggressive tumor characteristics, such as lymph node metastasis and larger tumors. In conclusion, the present study revealed that Lin28 expression served as a risk factor for lymph node metastasis. Accordingly, Lin 28 expression may be used as a prognostic marker to predict lymph node metastasis in patients with PTC. In addition, Lin28 may serve as a therapeutic target in the management of this tumor type, which may help improve patient outcomes.
\end{abstract}

Correspondence to: Dr Chaoyang Xu, Department of Breast and Thyroid Surgery, Affiliated Jiunhua Hospital, Zhejiang University School of Medicine, 365 Renmin East Road, Jiunhua, Zhejiang 321000, P.R. China

E-mail: xuchaoyang@zju.edu.cn

Key words: thyroid carcinoma, Lin28, lymph node stages, prognosis, clinicopathological parameters

\section{Introduction}

Thyroid cancer is uncommon, accounting for only $~ 1 \%$ of all malignancies, but it is the most common malignant endocrine tumor worldwide. Papillary thyroid carcinoma (PTC) accounts for $85 \%$ of thyroid malignancies and is defined as an indolent malignancy (1-3). Currently, the treatment for PTC is surgery, and either thyroid lobectomy or total thyroidectomy may be considered. Lymph node dissection may be performed in patients with evidence of nodal disease (4). Postoperative radioiodine therapy is required for patients at a high risk of cancer recurrence and persistent disease $(5,6)$. New therapies that target specific molecular alterations in PTC have been developed, for example lenvatinib is associated with significant improvements in progression-free survival and the response rate among patients with iodine-131-refractory thyroid cancer (7). In February 2015, the US FDA approved levatinib for the treatment of advanced thyroid cancer. Overall prognosis is not as poor in PTC as in several other human cancer types as the 5 -year survival rate of PTC is $>95 \%$. However, the standard therapy including surgery, and adjuvant radioactive iodine and thyroid-stimulating hormone (TSH) suppression therapy results in little clinical benefit for patients with lymph node or distant metastases. Thus, identification of new markers and potential treatment methods may be highly beneficial for these patients $(3,8)$.

Lin28 is a highly conserved RNA-binding and microRNA (miRNA/miR)-regulated protein. Recent studies have shown that Lin28 contributes to multiple biological processes, including tumorigenesis, cellular differentiation, pluripotency and reprogramming $(9,10)$. Lin28 expression is dysregulated in various human epithelial-type neoplasms, such as oral squamous cell carcinoma (11), colon (12), ovarian (13) and gastric cancer (14), hepatocellular carcinoma (15), and lung (16), breast (17) and esophageal cancer (18). Our previous studies have indicated that overexpression of Lin28 is correlated with lymph node metastasis and poor prognosis in breast (17) and gastric cancer (14). In general, Lin28 can selectively block the biogenesis of mature let-7, which acts as a tumor suppressor by inhibiting the expression of the oncogenes downstream of the Lin28/let-7 axis, including RAS, Myc and high mobility group protein HMGI-C (19). Let-7 miRNAs are downregulated in 
numerous types of cancer, such as hepatocellular carcinoma, gastric adenocarcinoma, pancreatic ovarian and prostate cancer, Burkitt lymphoma, renal cell carcinoma and breast cancer, and can suppress the proliferation of self-renewing tumor-initiating cells and facilitate differentiation (20). To the best of our knowledge, only one study has investigated the relationship between Lin28 and PTC (21). However, the number of clinical samples used in this previous study was very low, with only 57 patients after thyroid carcinoma radical surgery and 30 patients with nodular goiters. Moreover, this group investigated the role of the Lin28a/let-7a/c-Myc pathway in PTC tumorigenesis and malignancy but did not investigate the relationship between Lin28 expression and clinicopathological parameters. The objective of the current study was to analyze the association between the clinicopathological parameters and prognosis of PTC, whereby new preventive or therapeutic targets for PTC may be identified.

\section{Materials and methods}

Case selection. For the purpose of this study, a total of 237 surgically resected specimens of patients with PTC treated at the Department of Breast and Thyroid Surgery, Shaoxing People's Hospital (Shaoxing, China) between January 2016 and December 2018 were collected. The inclusion criteria for the study presented were as follows: i) Patients who had complete clinical data and had undergone radical resection of thyroid carcinoma and ii) patients who were definitively diagnosed with PTC by two experienced pathologists at Shaoxing People's Hospital. The present study excluded patients unwilling to abide by the protocol, as well as those that were legally incapacitated. Patients who had either received radiotherapy or chemotherapy prior to the surgical resection procedure were also excluded. In total, 237 patients were included in the study, including 47 males (19.83\%) and 190 females (80.17\%) aged 19-75 years, with an average age of 47.4 years. The original data (with respect to the specimens) are provided in Table SI. All patients signed informed consent under the guidelines of The Ethics Committee of Shaoxing People's Hospital before the study. The records reviewed included age, sex, tumor size, lymph node metastasis, bilateral multifocality, extrathyroidal extension and Lin28 expression level. Pathological features were classified according to the guidelines of the International Union against Cancer and the 8th edition of the American Joint Committee (22).

Ethics approval. The study was approved by The Ethics Committee of the Shaoxing People's Hospital (Shaoxing, China) before initiation. Written informed consent or verbal informed consent via phone (which was recorded) was provided by all participants or their guardians. These two forms of consent are both acceptable by the Ethics Committee of The Shaoxing People's Hospital. The consent forms can be provided upon reasonable request.

Immunohistochemical (IHC) analysis. IHC analysis was performed to evaluate the expression levels of the Lin 28 protein in PTC tissues. The specimens were fixed with formaldehyde and embedded in paraffin, as previously described (18). Paraffin sections were deparaffinized in xylene and dehydrated with a gradient ethanol series with 5-min washes at room temperature as follows: 100, 100, 95, 95, 80, 70 and 50\% ethanol, followed by two washes with $\mathrm{ddH}_{2} \mathrm{O}$. To quench the activity of endogenous peroxidase, the sections were treated with $0.3 \% \mathrm{H}_{2} \mathrm{O}_{2}$ in methanol for $5 \mathrm{~min}$. Before starting IHC analysis, the sections were immersed in $10 \mathrm{mM}$ citrate buffer $(\mathrm{pH}=6.1)$ at $95^{\circ} \mathrm{C}$ for $15 \mathrm{~min}$ for antigen retrieval. Non-specific antibody binding was blocked by pre-incubating the sections with $10 \%$ fetal calf serum (Gibco; Thermo Fisher Scientific, Inc.) in PBS with $0.01 \%$ sodium azide at room temperature for $30 \mathrm{~min}$, and then the sections were incubated with an antibody against Lin28 (rabbit polyclonal, H-44, 1:100; Santa Cruz Biotechnology Inc.) overnight at $4^{\circ} \mathrm{C}$. After washing three times with PBS, the sections were incubated with the EnVision-HRP complex (undiluted; Dako; Agilent Technologies, Inc.) for $1 \mathrm{~h}$ at room temperature. The sections were then stained with diaminobenzidine at room temperature for $25 \mathrm{sec}$ and counterstained with hematoxylin at room temperature for $5 \mathrm{~min}$. PBS, instead of Lin28, was used as a negative control. Gastric cancer tissue, which is known to exhibit high levels of Lin28 (19), was used as a positive control.

All the IHC analysis steps were carried out in the same laboratory. Expression levels were semi-quantitatively assessed based on the product of the cell staining intensity score and positive cell percentage score. The staining results were determined by two independent observers to avoid subjective biases. Staining intensity, which represented the average intensity of the stained tumor cells, was scaled as 0 (no staining), 1 (weak staining), 2 (moderate staining) and 3 (strong staining). Scores of percent positive cells were as follows: 0 points for positive rate $=0 \%, 1$ point for $0 \%<$ positive rate $\leq 25 \%, 2$ points for $25 \%$ $<$ positive rate $\leq 50 \%, 3$ points for $50 \%<$ positive rate $\leq 75 \%$, $75 \%<$ positive rate $\leq 100 \%$ is 4 points. The two scores were calculated to obtain a final score, which ranged from 0 to 9 . Three visual fields were randomly selected for each specimen under a light microscope, and their average values were applied for the final analysis. Lin 28 protein-positive expression was defined as total score $\geq 2$.

Bioinformatics analysis. Gene expression data and corresponding clinical data were obtained from the Gene Expression Omnibus (GEO) database (ncbi.nlm.nih.gov/gds). The dataset GSE33630 (23) was extracted from the GEO database was analyzed using a paired student's t-test to compare the differential expression levels of Lin28 between PTC and normal thyroid tissues. These databases were searched for data on Lin28 expression and lymph node metastasis, but no appropriate data was found. The human gene expression array GPL15207 (24) was downloaded from the GEO database. Three different groups were included in the study: i) Non-aggressive tumor (intrathyroidal tumors, with no lymphovascular invasion) with carcinoma sizes $<2 \mathrm{~cm}$ (group $\mathrm{A}, \mathrm{n}=3$ ), ii) patients with minimal invasion of the extrathyroidal soft tissue (group $\mathrm{B}, \mathrm{n}=3$ ) and iii) patients with distant organ metastasis (group $\mathrm{C}, \mathrm{n}=3)$. The age distributions of the three groups were $47.50 \pm 7.44,47.00 \pm 16.16$, and $55.00 \pm 15.63$, respectively. The proportions of women are $75.0,86.4$, and $75.0 \%$. The gene expression data of $\operatorname{Lin} 28$ were extracted to perform one-way ANOVA followed by Tukey's post hoc test among three groups to explore whether Lin28 expression influences distant organ 

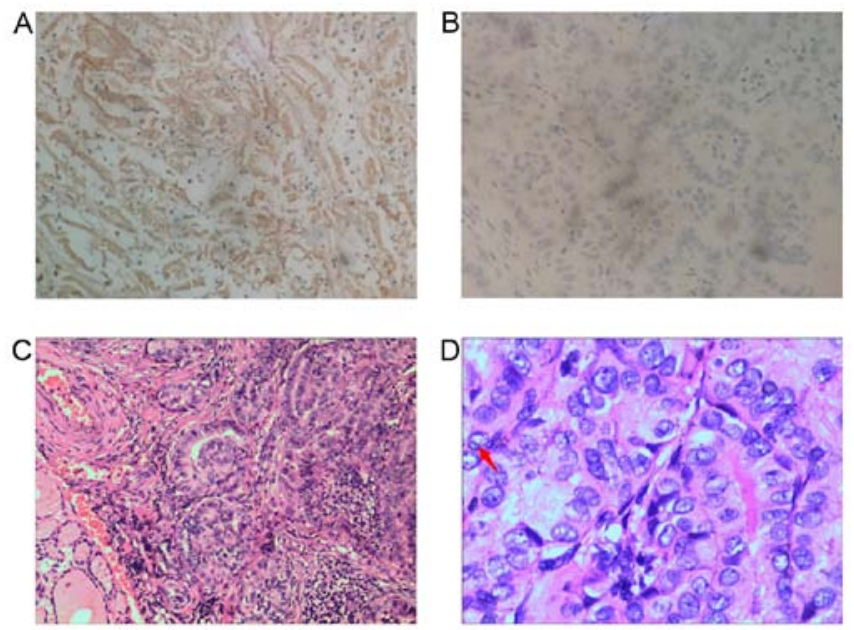

Figure 1. Lin28 expression profiling in thyroid carcinoma tissues. (A) Lin28-positive thyroid carcinoma cells, with cytoplasmic staining (200x magnification). (B) Lin28-negative thyroid carcinoma cells, with no cytoplasmic staining (200x magnification). Representative haematoxylin and eosin staining of papillary thyroid carcinoma at (C) 200x and (D) 400x magnification.

metastasis or invasion of the extrathyroidal soft tissue of patients with PTC.

Statistical analysis. All the statistical analyses were conducted using SPSS version 15.0. (SPSS, Inc). Quantitative data is expressed as mean \pm SD. The unpaired Student's t-test and $\chi^{2}$ test were performed to evaluate the statistical significance of the differences between two groups and the association between Lin28 expression and clinicopathological parameters, respectively. One-way ANOVA and Tukey's test was used for multiple comparisons. The predictive values of the presence of Lin 28 and other factors for lymph node metastases were assessed using univariate and multivariate logistic regression models, respectively. All the variables that were significantly associated with lymph node metastasis in the univariate model were included in the multivariate analysis. $\mathrm{P}<0.05$ was considered to indicate a statistically significant difference.

\section{Results}

Expression profiling of Lin28 protein in tissues. Lin28 protein was expressed in 96/237 (40.5\%) PTC specimens. Lin28 staining was predominantly localized in the cytoplasm in these tissues (Fig. 1A). A representative PTC specimen stained with hematoxylin and eosin is shown in Fig. 1C and D. It shows the nuclear features of papillary thyroid carcinoma, including crowded and overlapping grooves and pseudo-inclusions.

Association between Lin28 expression and clinicopathological features. Table I shows the association between Lin28 expression levels and clinicopathological characteristics, including age, sex, tumor size, lymph node metastasis, bilateral multifocality and extrathyroidal extension. Statistical analysis results showed that Lin28-positive cases had significantly larger tumors $(\mathrm{P}=0.011)$ and more lymph node metastases compared Lin28-negative cases $(\mathrm{P}<0.001)$. However, no difference was observed between the two groups with regards
Table I. Clinicopathological characteristics of 237 patients with papillary thyroid carcinoma.

\begin{tabular}{|c|c|c|c|}
\hline \multirow[b]{2}{*}{ Variable } & \multicolumn{2}{|c|}{ Lin28 expression, $\mathrm{n}(\%)$} & \multirow[b]{2}{*}{ P-value } \\
\hline & Positive & Negative & \\
\hline Age, years & $47.54 \pm 10.6$ & $47.27 \pm 11.8$ & 0.859 \\
\hline Sex & & & 0.990 \\
\hline Female & $77(80.2)$ & $113(80.1)$ & \\
\hline Male & $19(19.8)$ & $28(19.9)$ & \\
\hline Tumor size, $\mathrm{cm}$ & & & 0.011 \\
\hline$\leq 2$ & $87(90.6)$ & $135(95.7)$ & \\
\hline$>2$ and $\leq 4$ & $6(6.2)$ & $5(3.5)$ & \\
\hline$>4$ & $3(3.1)$ & $1(0.7)$ & \\
\hline Lymph node metastasis & & & $<0.001$ \\
\hline Yes & $73(30.8)$ & $35(14.8)$ & \\
\hline No & $23(9.7)$ & $106(44.7)$ & \\
\hline Multiple foci & & & 0.647 \\
\hline Yes & $21(21.9)$ & $19(13.5)$ & \\
\hline No & $75(78.1)$ & $122(86.5)$ & \\
\hline Extrathyroidal extension & & & 0.441 \\
\hline Yes & $6(6.3)$ & $12(8.5)$ & \\
\hline No & $90(93.8)$ & $129(91.5)$ & \\
\hline
\end{tabular}

to sex, age, or the presence of bilateral multifocality or extrathyroidal extension (Table I).

Multivariate analysis. A logistic regression model was constructed for the factors associated with lymph node metastasis in PTC (Table II). Sex (odds ratio [OR] 0.44; $\mathrm{P}=0.015$ ), tumor size (OR 3.46; $\mathrm{P}<0.001$ ) and the presence of $\mathrm{Lin} 28$ (OR 9.61; $\mathrm{P}<0.001)$ were associated with lymph node metastasis in the univariate analysis. These parameters were then analyzed using multivariate analysis. The results showed that sex, tumor size and the presence of Lin28 were risk factors for lymph node metastasis in PTC (Table II). The presence of Lin28 was found to be a risk factor for lymph node metastasis in PTC.

RNA expression levels of Lin28 in PTC according to GEO and TCGA databases. In the GEO database, Lin28A expression was slightly elevated in PTC tissues compared with the levels in normal thyroid tissue, but without statistical significance ( $\mathrm{P}=0.2022$; Fig. S1A). In addition, data referring to PTC patients' lymph node metastasis could not be obtained from both TCGA and GEO databases. However, data on distant organ metastasis in the context of PTC were obtained from the GEO database (Fig. S1B and C). It was revealed that Lin28 is associated with aggressive characteristics for patients with PTC, but this was not significant.

\section{Discussion}

As the most common type of thyroid carcinoma, PTC has received increasing attention because of its increasing morbidity rate worldwide (1). It is estimated that there were 567,233 new 
Table II. Results of multivariate analysis for the risk factors of lymph node metastases in papillary thyroid carcinoma $(\mathrm{n}=237)$.

\begin{tabular}{|c|c|c|c|c|}
\hline \multirow[b]{2}{*}{ Variable } & \multicolumn{2}{|c|}{ Univariate analysis } & \multicolumn{2}{|c|}{ Multivariate analysis } \\
\hline & OR $(95 \% \mathrm{CI})$ & P-value & OR $(95 \% \mathrm{CI})$ & P-value \\
\hline Lin28 expression, positive vs. negative & $9.61(5.25-17.60)$ & $<0.001$ & $10.16(5.25-19.64$ & $<0.001$ \\
\hline Sex, female vs. male & $0.44(0.23-0.85)$ & 0.015 & $0.37(0.16-0.82)$ & 0.015 \\
\hline Tumor size ${ }^{\mathrm{a}}, \mathrm{cm}$ & $3.46(2.02-5.94)$ & $<0.001$ & $3.16(1.73-5.77)$ & $<0.001$ \\
\hline $\operatorname{Age}^{\mathrm{a}}$, years & $0.99(0.97-1.01)$ & 0.288 & - & - \\
\hline Multiple foci, multifocal vs. unifocal & $1.05(0.49-2.27)$ & 0.897 & - & - \\
\hline Extrathyroidal extension, yes vs. no) & $1.13(0.77-1.66)$ & 0.538 & - & - \\
\hline
\end{tabular}

${ }^{a}$ Continuous variables. Others were categorized variables. OR, odds ratio; CI, confidence interval.

cases of thyroid cancer worldwide in 2018 (25). Benefiting from considerable therapeutic development, PTC prognosis and overall survival time have been associated with significant improvements (26). In PTC, metastasis to lymph nodes is associated with an increased risk of cancer recurrence but not with survival $(3,27,28)$. Additionally, extranodal extension of lymph node metastases is associated with a significantly increased risk of recurrence and mortality $(29,30)$. However, some patients with PTC have poor overall survival due to the presence of distant metastases at diagnosis $(31,32)$. Consequently, it is important to identify novel biological targets that can be used as prognostic markers for patients with PTC.

Numerous cancer-related genes are closely correlated with PTC progression. These genes affect the pathogenesis and prognosis of PTC. For example, Siraj et al (33) demonstrated a strong relationship between DNA (cytosine-5)-methyltransferase $3 \mathrm{~A}$ mutations, and adverse clinical outcomes in PTC cases. Previous studies have reported that telomerase reverse transcriptase promoter mutations have an independent prognostic value in differentiated thyroid carcinomas and in PTC $(32,34)$. In addition, Collina et al $(35)$ reported that tyrosine-protein kinase receptor UFO levels can be used as a predictor of radioactive iodine refractoriness and as a possible therapeutic target for radioactive iodine-resistant PTCs. Han et al (36) demonstrated that miR-215 plays a critical role as a tumor suppressor in PTC and is a prognostic biomarker influencing proliferation and metastasis by targeting the ADP ribosylation factor guanine nucleotide-exchange factor 1. Taken together, these prior studies have highlighted the importance of the involvement of genes in cancer development.

Lin28 expression has been widely profiled in multiple cancer types, such as glioblastoma and ovarian, gastric, prostate and breast cancer, and most studies have shown that Lin28 serves as a marker for poor survival in patients $(19,37,38)$. Lin 28 can promote the proliferation, migration and invasion of various human epithelial-type neoplasms, and might be a promising target for therapeutic intervention in the future (19). However, limited investigation has been conducted on the relationship between PTC and Lin28. The only such investigation to date, carried out by Huang et al (21), reported that the Lin28A/let-7a/c-Myc pathway is involved in tumor growth and malignancy in PTC and is a potential target for therapeutic intervention for this disease.
The present study attempted to provide a further prognostic basis regarding the association between Lin28 expression and PTC. Therefore, Lin28 expression patterns in PTC specimens were preliminarily investigated. It was demonstrated that Lin28 expression tends to be associated with more extensive lymph node metastasis in PTC. To validate the present results, additional data was retrieved and analyzed from the GEO online database. However, the results obtained for our clinical samples were not reproduced. A possible explanation for the partial non-reproducibility of the results reported in the present study might be due to the limited data on PTC and Lin28 available in the online databases, especially considering the presence of lymph node metastasis.

In future research, the molecular mechanisms and pathways underlying the involvement of Lin 28 in PTC should be investigated. In addition, the present results should be verified using data retrieved from additional online databases, depending on availability. It is considered that with a higher number of datasets, the data will align with what is reported in the present study. This said, well-designed studies including animal models and studies with larger ample sizes will also be needed to validate the present observations.

In summary, Lin28 expression was markedly elevated in PTC tissues. In addition, the expression of the Lin28 protein could be used as a novel molecular marker for predicting the clinical outcome of PTC from specimens. Hence, future investigations on the precise roles of Lin28 in PTC will enhance our understanding of this disease and provide new insights into its diagnosis and treatment.

\section{Acknowledgements}

Not applicable.

\section{Funding}

The study was supported by grants from The National Natural Science Foundation of China (grant no. 81341135), Zhejiang Non-profit Technology-Applied Research Projects of China (grant no. 2016C33226) and Shaoxing Non-profit Technology-Applied Research Projects of China (grant no. 2017B70037 2017QN002). 


\section{Availability of data and materials}

The datasets used and/or analyzed during the current study are available from the corresponding author upon reasonable requests. The datasets generated and/or analyzed during the current study are available in The Cancer Genome [cancergenome.nih.gov] and Gene Expression Omnibus repositories [ncbi.nlm.nih.gov/gds].

\section{Authors' contributions}

CX, LW and SJ conceived and directed the project. JW provided the pathological sections and performed the immunochemical scoring together with SW. CX and SJ collected, analyzed and interpreted the clinical data. SJ drafted and finalized the manuscript. All the authors read and approved the final version of the manuscript.

\section{Ethics approval and consent to participate}

The study protocols were approved by The Ethics Committee of the Shaoxing People's Hospital (Shaoxing, China; approval no. 2020-K-Y-079-01). All the participants or their guardians provided written informed consent.

\section{Patient consent for publication}

Not applicable.

\section{Competing interests}

The authors declare that they have no competing interests.

\section{References}

1. Kitahara CM and Sosa JA: The changing incidence of thyroid cancer. Nat Rev Endocrinol 12: 646-653, 2016.

2. Fagin JA and Wells SA Jr: Biologic and clinical perspectives on thyroid cancer. N Engl J Med 375: 2307, 2016.

3. Rosai J, Albores Saavedra J, Asioli S, et al: Papillary thyroid carcinoma. In: WHO Classification of Tumors of Endocrine Organs. Lloyd RV, Osamura RY, Klöppel G and Rosai J (eds). 4th edition. IARC, Lyon, 2017.

4. Ito Y, Higashiyama T, Takamura Y, Miya A, Kobayashi K, Matsuzuka F, Kuma K and Miyauchi A: Risk factors for recurrence to the lymph node in papillary thyroid carcinoma patients without preoperatively detectable lateral node metastasis: Validity of prophylactic modified radical neck dissection. World J Surg 31: 2085-2091, 2007.

5. Cooper DS, Doherty GM, Haugen BR, Kloos RT, Lee SL, Mandel SJ, Mazzaferri EL, McIver B, Pacini F Schlumberger M, et al: Revised American Thyroid Association management guidelines for patients with thyroid nodules and differentiated thyroid cancer. Thyroid 19: 1167-1214, 2009.

6. Francis GL, Waguespack SG, Bauer AJ, Angelos P, Benvenga S, Cerutti JM, Dinauer CA, Hamilton J, Hay ID, Luster M, et al: Management guidelines for children with thyroid nodules and differentiated thyroid cancer. Thyroid 25: 716-759, 2015.

7. Schlumberger M, Tahara M, Wirth LJ, Robinson B, Brose MS, Elisei R, Habra MA, Newbold K, Shah MH, Hoff AO, et al: Lenvatinib versus placebo in radioiodine-refractory thyroid cancer. N Engl J Med 372: 621-630, 2015.

8. Khatami F, Larijani B, Nikfar S, Hasanzad M, Fendereski K and Tavangar SM: Personalized treatment options for thyroid cancer: Current perspectives. Pharmgenomics Pers Med 12: 235-245, 2019.

9. Li M, Chen $\mathrm{H}$ and Wu T: LIN28: A cancer stem cell promoter for immunotherapy in head and neck squamous cell carcinoma. Oral Oncol 98: 92-95, 2019.
10. Liu Y, Dong N, Li J, Zhao L, Gao L, Zhang Y and Ruan J: RNA-binding protein Lin28 is associated with injured dentin-dental pulp complex in Sprague-Dawley rats. Int J Clin Exp Pathol 11: 4385-4394, 2018.

11. Wu J, Zhao W, Wang Z, Xiang X, Zhang S and Liu L: Long non-coding RNA SNHG20 promotes the tumorigenesis of oral squamous cell carcinoma via targeting miR-197/LIN28 axis. J Cell Mol Med 23: 680-688, 2019.

12. Zhang H, Zong Y, Qiu G, Jia R, Xu X, Wang F and Wu D: Silencing Lin 28 promotes apoptosis in colorectal cancer cells by upregulating let7c targeting of antiapoptotic BCL2L1. Mol Med Rep 17: 5143-5149, 2018.

13. He Y, Wang H, Yan M, Yang X, Shen R, Ni X, Chen X, Yang P, Chen M, Lu X, et al: High LIN28A and PLK4 coexpression is associated with poor prognosis in epithelial ovarian cancer. Mol Med Rep 18: 5327-5336, 2018.

14. Xu C, Shen J, Xie S, Jiang Z, Huang L and Wang L: Positive expression of Lin28 is correlated with poor survival in gastric carcinoma. Med Oncol 30: 382, 2013.

15. McDaniel K, Hall C, Sato K, Lairmore T, Marzioni M, Glaser S, Meng F and Alpini G: Lin28 and let-7: Roles and regulation in liver diseases. Am J Physiol Gastrointest Liver Physiol 310: G757-G765, 2016.

16. Yang Y, Li H, Liu Y, Chi C, Ni J and Lin X: MiR-4319 hinders YAP expression to restrain non-small cell lung cancer growth through regulation of LIN28-mediated RFX5 stability. Biomed Pharmacother 115: 108956, 2019.

17. Xu C, Jin S and Huang L: Expression of Lin28 is correlated with prognosis and expression of HER-2 and steroid receptors in breast cancer. Onco Targets Ther 12: 1105-1110, 2019.

18. Ling R, Zhou Y, Zhou L, Dai D, Wu D, Mi L, Mao C and Chen D: Lin 28/microRNA-let-7a promotes metastasis under circumstances of hyperactive Wnt signaling in esophageal squamous cell carcinoma. Mol Med Rep 17: 5265-5271, 2018.

19. Balzeau J, Menezes MR, Cao S and Hagan JP: The LIN28/let-7 pathway in cancer. Front Genet 8: 31, 2017.

20. Ji J and Wang XW: A Yin-Yang balancing act of the lin28/let-7 link in tumorigenesis. J Hepatol 53: 974-975, 2010.

21. Huang J, Lin H, Zhong M, Huang J, Sun S, Lin L and Chen Y: Role of Lin28A/let-7a/c-Myc pathway in growth and malignant behavior of papillary thyroid carcinoma. Med Sci Monit 24: 8899-8909, 2018.

22. Amin MB, Greene FL, Edge SB, Compton CC, Gershenwald JE, Brookland RK, Meyer L, Gress DM, Byrd DR and Winchester DP: The Eighth edition AJCC Cancer Staging Manual: Continuing to build a bridge from population-based to a more 'personalized' approach to cancer staging. CA Cancer J Clin 67: 93-99, 2017.

23. Dom G, Tarabichi M, Unger K, Thomas G, OczkoWojciechowska M, Bogdanova T, Jarzab B, Dumont JE, Detours V and Maenhaut C: A gene expression signature distinguishes normal tissues of sporadic and radiation-induced papillary thyroid carcinomas. Br J Cancer 107: 994-1000, 2012.

24. Akyay OZ, Gov E, Kenar H, Arga KY, Selek A, Tarkun I, Canturk Z, Cetinarslan B, Gurbuz Y and Sahin B: Mapping the molecular basis and markers of papillary thyroid carcinoma progression and metastasis using global transcriptome and microRNA profiling. OMICS 24: 148-159, 2020.

25. Bray F, Ferlay J, Soerjomataram I, Siegel RL, Torre LA and Jemal A: Global cancer statistics 2018: GLOBOCAN estimates of incidence and mortality worldwide for 36 cancers in 185 countries. CA Cancer J Clin 68: 394-424, 2018.

26. He J, Zhou M, Li X, Gu S, Cao Y, Xing T, Chen W, Chu C, Gu F, Zhou J, et al: SLC34A2 simultaneously promotes papillary thyroid carcinoma growth and invasion through distinct mechanisms. Oncogene 39: 2658-2675, 2020.

27. Loh KC, Greenspan FS, Gee L, Miller TR and Yeo PP: Pathological tumor-node-metastasis (pTNM) staging for papillary and follicular thyroid carcinomas: A retrospective analysis of 700 patients. J Clin Endocrinol Metab 82: 3553-3562, 1997.

28. Guo K and Wang Z: Risk factors influencing the recurrence of papillary thyroid carcinoma: A systematic review and metaanalysis. Int J Clin Exp Pathol 7: 5393-5403, 2014.

29. Veronese N, Luchini C, Nottegar A, Kaneko T, Sergi G, Manzato E, Solmi M and Scarpa A: Prognostic impact of extra-nodal extension in thyroid cancer: A meta-analysis. J Surg Oncol 112: 828-833, 2015.

30. Chereau N, Buffet C, Tresallet C, Tissier F, Leenhardt L and Menegaux F: Recurrence of papillary thyroid carcinoma with lateral cervical node metastases: Predictive factors and operative management. Surgery 159: 755-762, 2016. 
31. Su DH, Chang SH and Chang TC: The impact of locoregional recurrences and distant metastases on the survival of patients with papillary thyroid carcinoma. Clin Endocrinol (Oxf) 82: 286-294, 2015.

32. Melo M, Gaspar da Rocha A, Batista R, Vinagre J, Martins MJ, Costa G, Ribeiro C, Carrilho F, Leite V, Lobo C, et al: TERT, BRAF, and NRAS in primary thyroid cancer and metastatic disease. J Clin Endocrinol Metab 102: 1898-1907, 2017.

33. Siraj AK, Pratheeshkumar P, Parvathareddy SK, Bu R, Masoodi T, Iqbal K, Al-Rasheed M, Al-Dayel F, Al-Sobhi SS, Alzahrani AS, et al: Prognostic significance of DNMT3A alterations in Middle Eastern papillary thyroid carcinoma. Eur J Cancer 117: 133-144, 2019.

34. Melo M, da Rocha AG, Vinagre J, Batista R, Peixoto J, Tavares C, Celestino R, Almeida A, Salgado C, Eloy C, et al: TERT promoter mutations are a major indicator of poor outcome in differentiated thyroid carcinomas. J Clin Endocrinol Metab 99: E754-E765, 2014.
35. Collina F, La Sala L, Liotti F, Prevete N, La Mantia E, Chiofalo MG, Aquino G, Arenare L, Cantile M, Liguori G, et al: AXL is a novel predictive factor and therapeutic target for radioactive iodine refractory thyroid cancer. Cancers (Basel) 11: 785, 2019.

36. Han J, Zhang M, Nie C, Jia J, Wang F, Yu J, Bi W, Liu B, Sheng R, He G, et al: miR-215 suppresses papillary thyroid cancer proliferation, migration, and invasion through the AKT/GSK-3 $\beta /$ Snail signaling by targeting ARFGEF1. Cell Death Dis 10: 195, 2019.

37. Zhang J, Xu A, Miao C, Yang J, Gu M and Song N: Prognostic value of Lin28A and Lin28B in various human malignancies: A systematic review and meta-analysis. Cancer Cell Int 19: 79, 2019.

38. Viswanathan SR, Powers JT, Einhorn W, Hoshida Y, Ng TL, Toffanin S, O'Sullivan M, Lu J, Phillips LA, Lockhart VL, et al: Lin 28 promotes transformation and is associated with advanced human malignancies. Nat Genet 41: 843-848, 2009. 